\title{
A influência da osteoporose na implantodontia
}

\author{
The influence of osteoporosis on implant dentistry \\ La influencia de la osteoporosis en la implantología
}

Recebido: 05/1/2021 | Revisado: 11/11/2021 | Aceito: 11/11/2021 | Publicado: 15/11/2021

\author{
Pedro Henrique Ribeiro Mota \\ ORCID: https://orcid.org/0000-0002-2900-9629 \\ Universidade Brasil, Brasil \\ E-mail: pedroribeiromota101 @gmail.com \\ Caroline Liberato Marchiolli \\ ORCID: https://orcid.org/0000-0001-8881-4882 \\ Universidade Brasil, Brasil \\ E-mail: caaliberato@gmail.com \\ Maria Eduarda de Freitas Santana Oliveira \\ ORCID: https://orcid.org/0000-0002-4074-4288 \\ Universidade Brasil, Brasil \\ E-mail:mariaefreitas10@gmail.com \\ Fábio Roberto de Souza Batista \\ ORCID: https://orcid.org/0000-0002-5105-7686 \\ Universidade Estadual Paulista "Júlio de Mesquita Filho", Brasil \\ E-mail: fabiorsbatista@gmail.com
}

\begin{abstract}
Resumo
A osteoporose é uma patologia grave devido às fraturas de que é responsável. A frequência da doença é alta e proporcional à idade. A população brasileira está envelhecendo, portanto, está aumentando. O impacto da osteoporose no sistema de saúde irá, portanto, aumentar. Além de seu efeito no esqueleto em geral, a osteoporose também tem consequências significativas para a saúde bucal. Na verdade, pode ter um impacto: ao nível dos dentes, pode causar um afrouxamento gradual da dentição e a criação de espaços inter dentários; ao nível dos ossos da mandíbula, a perda óssea pode tornar mais difícil a adaptação de implantes dentários ou o ajuste de próteses. O envelhecimento da população e o envolvimento de certas doenças metabólicas que afetam o metabolismo ósseo pode interferir na colocação dos implantes. A osteoporose afeta a qualidade e a quantidade óssea, portanto a colocação do implante pode ser arriscada. Estudos associam a perda de implantes dentários e a osteoporose diretamente, embora não sejam estatisticamente significativos. Apesar do fato de que a osteoporose a quantidade e a qualidade os ossos são afetados, a formação óssea adequada ao redor do implante e com uma porcentagem semelhante de contato do osso com a superfície do implante foi demonstrada em indivíduos saudáveis versus indivíduos com osteoporose.
\end{abstract}

Palavras-chave: Osteoporose; Implantes dentários; Osseointegração; Fatores de risco; Reabsorção óssea.

\begin{abstract}
Osteoporosis is a serious condition due to the fractures it is responsible for. The frequency of the disease is high and proportional to age. The Brazilian population is aging, therefore, it is increasing. The impact of osteoporosis on the healthcare system will therefore increase. In addition to its effect on the skeleton in general, osteoporosis also has significant consequences for oral health. In fact, it can have an impact: at the level of the teeth, it can cause a gradual loosening of the dentition and the creation of interdental spaces; at the level of the jaw bones, bone loss can make it more difficult to fit dental implants or fit dentures. The aging of the population and the involvement of certain metabolic diseases that affect bone metabolism can interfere with implant placement. Osteoporosis affects bone quality and quantity, so implant placement can be risky. Studies link the loss of dental implants and osteoporosis directly, although they are not statistically significant. Despite the fact that osteoporosis bone quantity and quality are affected, adequate bone formation around the implant and with a similar percentage of bone contact with the implant surface has been demonstrated in healthy individuals versus individuals with osteoporosis.
\end{abstract}

Keywords: Osteoporosis; Dental implants; Osseointegration; Risk factors; Bone resorption.

\section{Resumen}

La osteoporosis es una patología grave por las fracturas que provoca. La frecuencia de la enfermedad es alta y proporcional a la edad. La población brasileña está envejeciendo, por lo que está aumentando. Por lo tanto, el impacto de la osteoporosis en el sistema sanitario aumentará. Además de su efecto sobre el esqueleto en general, la osteoporosis también tiene importantes consecuencias para la salud bucodental. De hecho, puede tener un impacto: a nivel de los dientes, puede provocar un aflojamiento gradual de la dentición y la creación de espacios interdentales; a nivel de los huesos de la mandíbula, la pérdida de hueso puede dificultar la colocación de implantes dentales o la colocación de 
prótesis. El envejecimiento de la población y la implicación de ciertas enfermedades metabólicas que afectan al metabolismo óseo pueden interferir en la colocación de los implantes. La osteoporosis afecta a la calidad y cantidad de hueso, por lo que la colocación de implantes puede ser arriesgada. Los estudios asocian directamente la pérdida de los implantes dentales y la osteoporosis, aunque no son estadísticamente significativos. A pesar de que la osteoporosis afecta a la cantidad y calidad del hueso, se demostró una formación ósea adecuada alrededor del implante con un porcentaje similar de contacto óseo con la superficie del implante en los sujetos sanos frente a los sujetos con osteoporosis.

Palabras clave: Osteoporosis; Implantes dentales; Osteointegración; Factores de riesgo; Resorción ósseo.

\section{Introdução}

A osteoporose se tornará um problema de saúde com uma escala global, pois a população está envelhecendo. A osteoporose é uma doença associada ao envelhecimento e, particularmente, às alterações hormonais associadas à menopausa. Portanto, é particularmente prevalente em mulheres na pós-menopausa, embora também possa afetar homens adultos e, em casos excepcionais, crianças (Costa, 2019).

A osteoporose é uma doença metabólica sistêmica que resulta em perda de massa óssea e defeitos da microarquitetura óssea. A análise da densidade óssea (densitometria óssea) é o exame reconhecido para detectar essa condição, mas um estudo recente em mulheres com osteoporose mostrou que essa patologia pode ser identificada em uma imagem dentária panorâmica usando índices radiomorfométricos como o Índice Cortical Mandibular (ICM) (Figura 1). Ele permite que os pacientes sejam divididos em três grupos: saudáveis, osteopênicos e osteoporóticos (Mesquita, 2010.)

Em estudos retrospectivos, sugere-se uma correlação significativa entre a osteoporose e o índice de ICM, entretanto, também há estudos em que a osteoporose, avaliada pelo Índice Cortical Mandibular, não parece estar correlacionada com o risco de perda óssea marginal. Esta perda óssea marginal parece estar correlacionada com uma história de periodontite e a colocação de implantes em um local de osso regenerado (Mesquita, 2010).

Figura 1. Obtenção dos índices morfométricos mandibulares, de acordo com os artigos originais que os preconizaram (10-13). $\mathrm{A}$, tangente à base da mandíbula; B, tangente à borda anterior do ramo; C, perpendicular a $\mathrm{A}$, na altura em que B cruza a base da mandíbula; D, tangente à borda posterior do ramo; E, bissetriz do ângulo formado pelas linhas A e D; F, perpendicular a A, na altura do centro do forame mentoniano. Os índices são obtidos, em mm, da seguinte maneira: IA - espessura da cortical mandibular sobre a linha C; IG - espessura da cortical mandibular sobre a linha E; IM - espessura da cortical mandibular sobre a linha F; IPM - razão da medida da espessura da cortical mandibular sobre a linha C pela distância entre a borda inferior da mandíbula e o limite inferior do canal mandibular sobre a mesma linha.

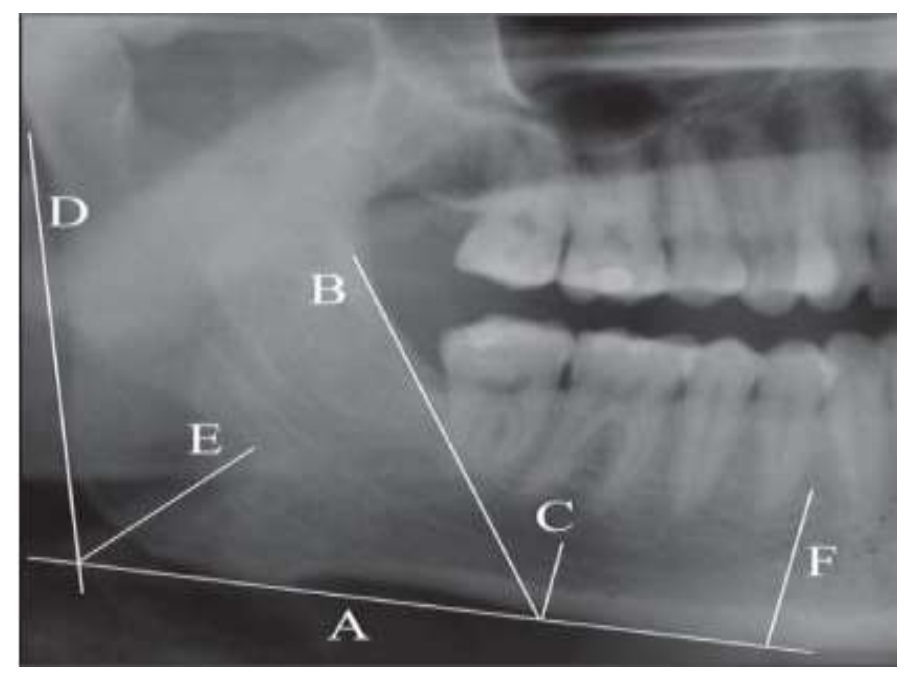

Fonte: Mahl et al. (2008) 
É importante salientar que o implante dentário para osteoporose é altamente recomendado sob certas condições. É necessário que o dentista tenha especial atenção e dedicação ao paciente com osteoporose, principalmente àquele que faz uso de bifosfonatos há muito tempo, que são os medicamentos comumente prescritos pelo médico no combate às perdas. As intervenções cirúrgicas devem ser restritas (Giro, et al., 2020).

Ao aplicar um implante dentário em um paciente com osteoporose, é essencial avaliar previamente as seguintes circunstâncias: há quanto tempo o paciente sofre da doença; há quanto tempo o paciente está tomando bifosfonatos (por via oral ou intravenosa) e quais são os hábitos do paciente no seu dia a dia, visto que alguns deles podem ter um efeito muito negativo nos implantes dentários.

Salienta-se apenas que os especialistas concordam de forma absolutamente unânime que o que se recomenda para os pacientes com osteoporose são os implantes dentários integrados ao osso, ou seja: os chamados implantes osseointegráveis. Estes constituem a melhor solução para implantes dentários com osteoporose. Entretanto, na verdade até o momento, não existem dados suficientes que contraindiquem a colocação de implantes em pacientes com osteoporose, é primordial analisar individualmente a situação de cada paciente e como profissional dentista avaliar todos os elementos e indicar se é viável ou não os implantes dentários (Maxuel, et al., 2020).

\section{Metodologia}

A pesquisa a ser realidade neste trabalho pode ser classificada como revisão bibliográfica qualitativa e descritiva inerente à temática proposta para o estudo. Os bancos de dados utilizados foram: Pubmed, Scielo, Lilacs, Google Acadêmico. Para auxílio na busca as palavras chave utilizadas foram: osteoporose, implantes dentários, osseointegração, fatores de risco, reabsorção óssea.

\section{Revisão de literatura}

De acordo com a definição da Organização Mundial de Saúde (OMS), a osteoporose é uma doença esquelética generalizada caracterizada por uma baixa densidade óssea e alterações na microarquitetura óssea (Figura 2), responsável pela fragilidade óssea exagerada e, portanto, um alto risco de fratura. "A densidade mineral óssea é o determinante essencial da fragilidade óssea. As fraturas são "a" complicação da doença osteoporótica e constituem todo a gravidade desta doença (Spezzia, 2017).

Figura 2. Micrografias comparando o osso normal (esquerda) com o osso osteoporótico (direita). Nota-se alteração na morfologia do trabeculado ósseo na osteoporose, sendo este, muito menos denso, tornando o osso mais suscetível a fratura. Retirado The American Society for Bone and Mineral Research, 1986.

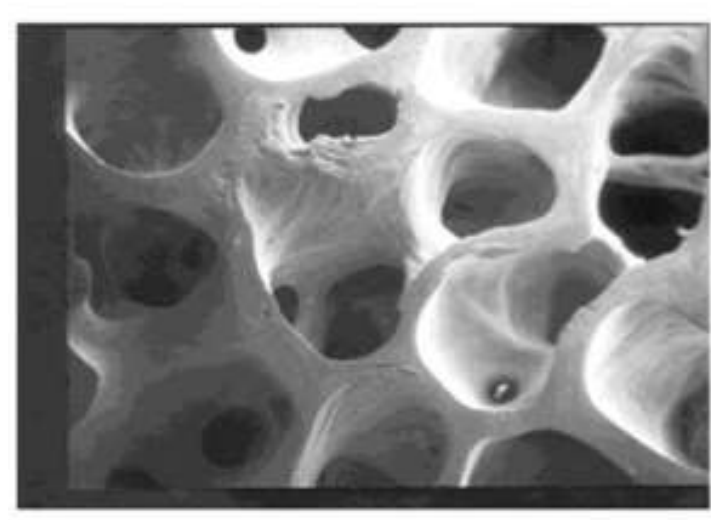

Osso Normal

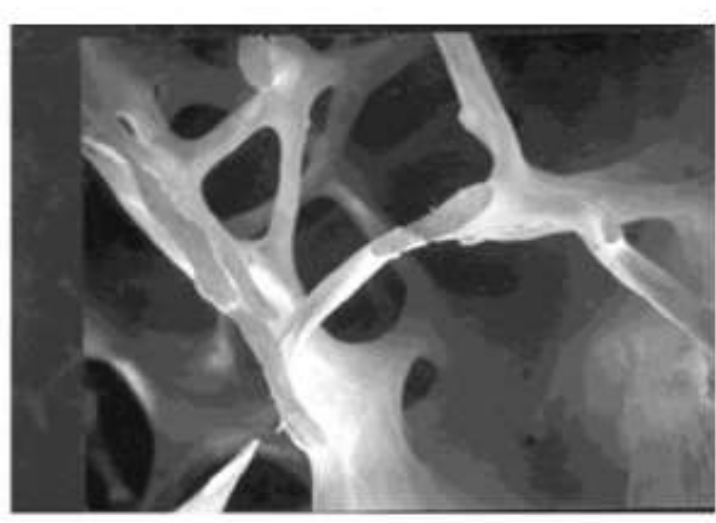

Osso Osteoporótico

Fonte: Bezerra (2017). 
De acordo com Luize et al., (2005), no momento da preparação do leito ósseo receptor para um implante osseointegrado, geralmente ocorre necrose óssea, que posteriormente esta deverá ser substituída por um novo tecido ósseo, este processo é considerado vital para a obtenção da osseointegração.

Os tratamentos para combater a osteoporose têm um impacto direto e indireto na saúde oral. Na verdade, as pessoas com osteoporose na maioria das vezes seguem um tratamento medicamentoso combinado de bisfosfonatos, muito eficaz no aumento da densidade mineral óssea e na redução do risco de contas e agente reabsorvente que inibe os fenômenos de reabsorção óssea. O principal efeito colateral do bifosfonato é a osteonecrose da mandíbula (OM), pois o mesmo causa degradação do osso da mandíbula, especialmente após cirurgia dentária. Nos consultórios odontológicos, uma porcentagem relativa, mostra que a osteonecrose da mandíbula é encontrada em pacientes tratados com bifosfonatos usados para prevenir a osteoporose (Gonçalves et al., 2020).

O dentista desempenha um papel de suma importância na descoberta, no tratamento e na prevenção da osteoporose, sugere-se que ao perceber sinais de osteoporose uma consulta dentaria o qual pode realizar alguns procedimentos como realizar a restauração da cavidade oral, pedir uma radiografia panorâmica com ICM, assim como oferecer um plano de tratamento e cuidados necessários para eliminar possíveis surtos de infecção, além de fornecer informações sobre os tratamentos e seus efeitos colaterais (Maxuel, et al., 2020).

$\mathrm{Na}$ implantodontia, uma obtenção de novo osso em torno da superfície do implante depende diretamente de uma população ativa e equilibrada de osteoblastos e osteoclastos. Qualquer perturbação nessa relação celular pode alterar a quantidade ou qualidade do osso formado, levando a um déficit de massa óssea (Gonçalves, et al., 2020).

Nessas condições, o processo de osseointegração está prejudicado em relação aos osteoporóticos. Geralmente as características biomecânicas do osso osteoporótico não são compatíveis com a estabilidade para implantes osseointegrados, sendo que alguns autores consideram o osso osteoporótico semelhante ao osso tipo IV, onde uma taxa de sucesso é claramente diminuída. Alguns estudos clínicos e experimentais têm discutido o risco de falhas de implantes osseointegrados em mulheres pós-menopausa com osteoporose. Infelizmente, ainda não foram avaliados dados suficientes que possam demonstrar os prejuízos causados pela deficiência sistêmica de estrógeno sobre o tecido ósseo, portanto, ainda não há uma contraindicação total sobre a colocação de implantes osseointegrados em mulheres (Nascimento, 2021).

No estudo de Luize et al., (2005) é citado um estudo realizado por Fujimoto et al., (1996), onde foi descrito um caso clínico de um paciente portadora de osteoporose severa que foi tratada com implantes dentais osseointegrados na mandíbula. O tratamento foi realizado de maneira convencional, exceto por um período maior de cicatrização (6 meses). Todos os implantes obtiveram osseointegração clínica e não houve qualquer problema após a instalação da sobre estrutura.

Estudos retrospectivos tentaram demonstrar os riscos oferecidos pela osteoporose no tratamento com implantes dentais, neles foram avaliados: as mudanças no conteúdo ósseo mineral em mandíbulas com overdentures implanto-suportadas; se as mudanças no conteúdo ósseo mineral se apresentava diferente em grupos sem e com barra conectando os implantes; se a presença de osteoporose mandibular (verificada com base na densidade óssea no início, 2 anos após e 5 anos após instalação do implante) afeta a perda de altura óssea ao redor de implantes (Luize, et al., 2005).

\subsection{Epidemiologia}

Em mulheres, a prevalência de osteoporose densitométrica ( $\mathrm{T}$-score $\leq-2,5)$ aumenta com a idade a partir dos 50 anos. É estimado em 39\% aos 65 anos e em torno70\% após 80 anos. A osteoporose afeta cerca de $40 \%$ das mulheres na pós-menopausa e $15 \%$ dos homens depois de 50 anos. A incidência de fraturas aumenta exponencialmente a partir dos 50 anos. Em humanos, a incidência de fraturas osteoporóticas é aproximadamente 3 vezes menor do que o observado nas mulheres (Nascimento, 2021). 


\subsection{Fisiopatologia (Fisiologia óssea)}

O tecido ósseo é um tecido conjuntivo especializado composto de células dispersas em uma abundante matriz extracelular. A matriz extracelular é composta por uma parte orgânica (fibras de colágeno tipo I e substância fundamental) e uma parte mineral (principalmente sais de cálcio). Esta matriz é rígida e mal hidratada: o tecido ósseo é composto por 50\% de água, o que o torna um dos tecidos menos hidratados depois do esmalte dental (Madrid \& Sanz, 2009).

Segundo Nascimento (2021), o tecido ósseo tem três funções:

- Um papel mecânico: o tecido ósseo é um dos tecidos mais resistentes emo organismo, capaz de suportar tensões mecânicas, dando o osso tem a função de sustentar o corpo e proteger os órgãos.

- Um papel metabólico: o tecido ósseo é um tecido dinâmico, constantemente remodelado sob o efeito de pressões mecânicas, resultando ema liberação ou armazenamento de sais minerais, garantindo assim em um em grande medida (juntamente com o intestino e os rins) o controle de metabolismo fosfocálcico.

- Um papel hematopoiético: os ossos contêm em seus espaços, medula hematopoiética, incluindo células-tronco, para a origem das três linhas de células do sangue, são encontradas nas proximidades células ósseas. Células estromais (células de tecido conjuntivo) na medula óssea fornecer suporte estrutural e funcional às células hematopoiético. Algumas delas são células tronco multipotente com probabilidade de se diferenciar em várias linhagens diferentes (fibroblastos, condrócitos, osteoblastos, adipócitos. As células do tecido ósseo incluem células especializadas em ósteo formação, osteoblastos e, na osteo ressorção, osteoclastos, também como osteócitos alojados em osteoplastos

Nos homens, uma osteoporose está relacionada ao declínio da testosterona, enquanto nas mulheres está interligada à deficiência de estrógeno. Essa osteoporose provocada pela deficiência do estrógeno ou da testosterona é chamada de osteoporose primária, sendo uma das mais específicas. A osteoporose, ocasionada pela redução dos níveis de estrógeno, afeta a mandíbula e a maxila assim como atinge os ossos longos. Os receptores de estrogênio têm sido encontrados nos ossos, afetando-os indiretamente por interagirem com hormônios que controlam o metabolismo do cálcio, dentre eles, os hormônios paratireoide, calcitonina e vitamina D (Almeida, 2019).

O uso dos estrógenos tem identificação de minimização na excreção urinária de cálcio e hidroxiapatita (um indicador de reabsorção óssea). Acredita-se que os estrógenos protegem os ossos da ação dos hormônios paratireoides, que é mediador da reabsorção óssea. O declínio desses hormônios acomete principalmente as mulheres no período da menopausa. Comprovadamente experimentos realizados em animais, verificam que a densidade mineral óssea é reduzida diante da deficiência desse hormônio (Camargos \& Bomfim, 2017).

A testosterona é um hormônio importante na regulação do funcionamento dos órgãos e tecidos corpóreos como os rins, coração, músculo estriado esquelético, sistema imunológico, glândulas salivares, tecidos orais e periodontais, como também, do tecido ósseo. As restrições do hormônio acabam diminuindo com o avançar da idade, e com esse decréscimo hormonal podem ocorrer mudanças no sistema cardiovascular, dislipidemia, hipertensão, síndrome metabólica, diabetes tipo 2, doença periodontal e osteoporose. Os homens não são poupados, no entanto. A osteoporose masculina relacionada à idade pode ser menos comum, não é trivial: um quarto das fraturas por fragilidade óssea ocorrem em homens (Almeida, 2019).

\subsection{Diagnóstico}

O diagnóstico precoce da osteoporose pode diminuir o risco de fraturas e melhorar a qualidade de vida dos indivíduos. Ao referir-se sobre o diagnóstico, a suspeita clínica deve ser investigada através da densidade mineral óssea (DMO) avaliada por meio de diversos exames, sendo considerada uma densitometria óssea de dupla emissão (Figura 3) (Varga \& Schimitt, 2016). 
Figura 3. Densitometria óssea de dupla emissão.
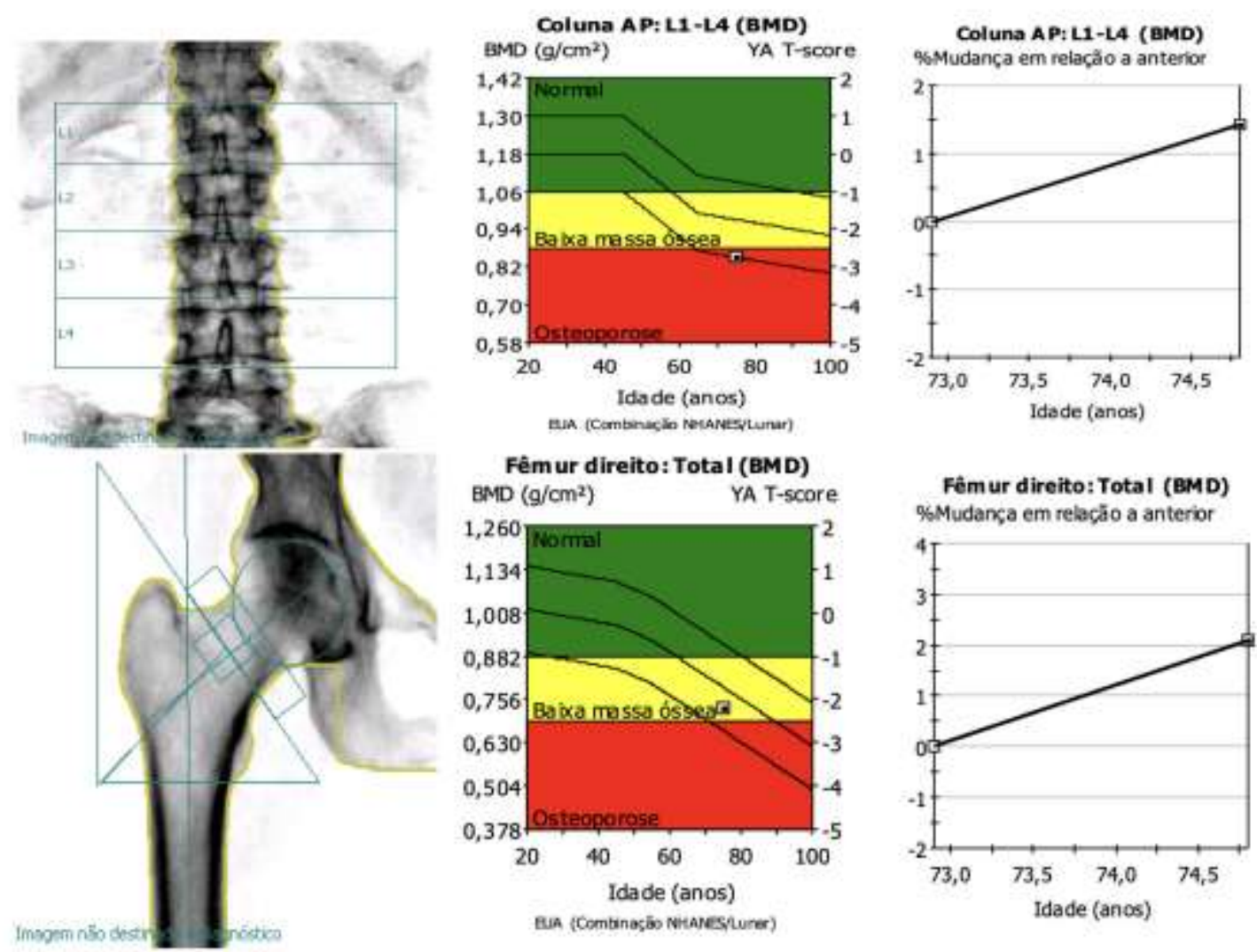

Fonte: Medicina Diagnóstica (2021).

Apesar da mesma, ser oferecida pelo Sistema Único de Saúde (SUS), a disponibilidade deste exame para a população ainda apresenta algumas dificuldades relacionadas, principalmente, ao seu custo elevado e ao número insuficiente de aparelhos. Desse modo, essa falha do sistema acaba por trazer não só um prejuízo no acesso ao exame, mas também, no diagnóstico precoce e tratamento da osteoporose. Em caso de osteoporose secundária o diagnóstico é realizado através da DMO, e também de exames laboratoriais que incluem hemograma completo, cálcio, fósforo, fosfatase alcalina, função tireoidiana, dosagem da $25(\mathrm{OH})$ vitamina D sérica, calciúria de 24 horas, além de radiografia da coluna torácica, lombar e fêmur proximal (Almeida, 2019),

Uma alternativa bem interessante tem sido uma opção alternativa no diagnóstico precoce da osteoporose, fato relacionado a um custo menor e ao elevado número de pacientes osteoporóticos, paciente das clínicas odontológicas. É benéfico o uso das radiografias panorâmicas para triagem da osteoporose não detectada. Outra vantagem do uso dessas radiografias panorâmicas é que costumam ser frequentemente recuperar, parâmetros de projeção e exposição semelhantes, tornando-as equipadas para comparação (Maxuel, et al., 2020).

Através do exame radiográfico é possível analisar os índices quantitativos, tais como, o Índice Mentual (IM), bem como os qualitativos desenvolvidos e validados, que permitem avaliar a arquitetura óssea com o objetivo de identificar sinais da osteoporose. Dentre os índices radiomorfométricos utilizados, tem-se o índice cortical mandibular, que é responsável por classificar a morfologia óssea da mandíbula cortical em três padrões: C1 (cortical sem alterações), C2 (erosão leve à moderada) e C3 (erosão severa), sendo os dois últimos padrões indicativos de osteoporose (Leite, et al., 2008). 


\subsubsection{O Papel das radiografias dentárias na triagem para osteoporose}

Os raios X são uma ferramenta de diagnóstico comum na odontologia hoje. Embora sejam indicados principalmente para diagnósticos odontológicos, eles também podem fornecer outras informações. Assim, raios-x dentais mostrando o osso mandibular ou maxilar também pode ser usado para diagnóstico de doenças ósseas (figura 4). Assim como na detecção de outras doenças da esfera orofacial, como carcinomas, o dentista pode cumprir o mesmo papel no diagnóstico possível osteoporose. É sugerido que o exame radiográfico do esqueleto facial pode levar a detecção precoce da osteoporose (Leite, et al., 2008).

Figura 4. A Radiografia panorâmica evidenciando osteólise peri-implantar e perda dos implantes. B. Tomografia computadorizada de mandíbula com aproximação comprovando a osteólise peri-implantar devido à presença de osteonecrose.

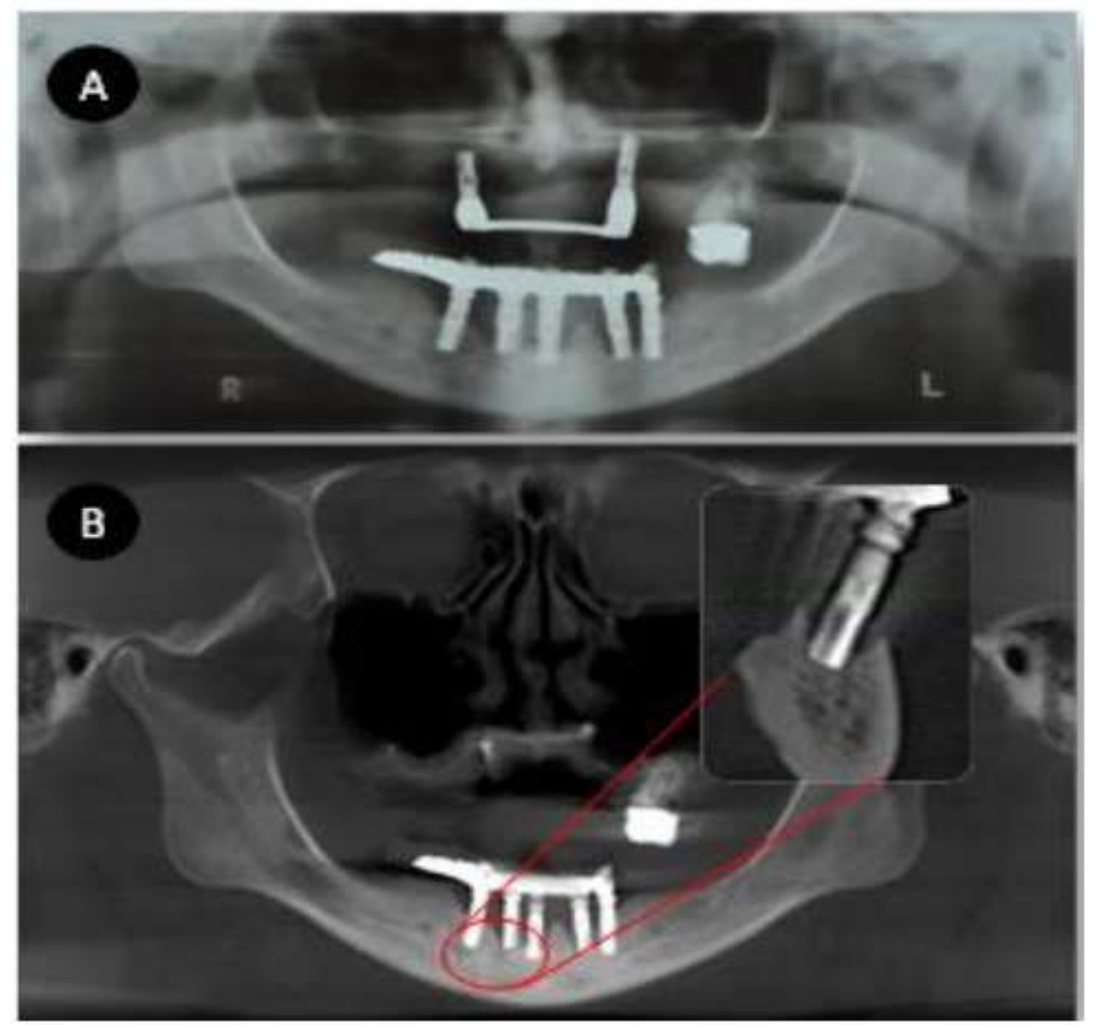

Fonte: Coléte, et al. (2019).

Segundo Nogueira et al., (2012), embora haja nenhum método padrão atual para medir a densidade mineral óssea da mandíbula e maxilar, novas tecnologias podem permitir aos dentistas avaliarem a qualidade do osso no nível oral. Quatro técnicas para estudar raios-x dentais foram comparadas com detectar osteoporose:

- A dimensão fractal: medida da perda de continuidade e dimensão de espaço trabecular;

- Microdensitometria: transmissão de luz através de uma área;

- Intensidade do pixel: preto ou branco;

- Análise panorâmica: espessura da cortical óssea no ângulo goníaco. Eles foram comparados a técnicas padrão para medir a densidade óssea usado para diagnosticar a osteoporose além dos ossos da mandíbula (absortometria de dois fótons e tomografia computadorizada quantitativa).

A intensidade do pixel é o método diagnóstico, de radiografia dentária mais eficiente. Raios X digitalizados podem ser um método mais confiável de monitoramento das mudanças na densidade óssea. Radiografias dentárias panorâmicas podem ser úteis na identificação em mulheres com menos de 65 anos têm osteoporose ao observar um córtex osso fino e/ou osso cortical 
severamente erodido. Estudos indicam que a densidade óssea da mandíbula é estatisticamente significativamente menor em pacientes com osteoporose (Souza, 2010).

No entanto, quando as pistas extraídas de Radiografias panorâmicas são usadas para correlacionar a densidade mineralosso da mandíbula ao do quadril ou punho, verifica-se que não são capazes de distinguir mandíbulas normais de mandíbulas osteoporóticas/osteopênica. A avaliação de radiografias panorâmicas odontológicas para a identificação das alterações osteoporóticas geralmente mostra resultados mistos (Leite, et al., 2008).

Em um estudo citado por Mesquita et al., (2010), uma radiografia dentária panorâmica em pacientes com osteoporose era mais propensa a ter uma mudança na morfologia corporal osso cortical inferior mandibular, mas nenhuma diferença foi encontrada estatisticamente significativa na largura do córtex, o grau de reabsorção da crista alveolar, a dimensão fractal ou o número de dentes inferiores, porém comparação com pacientes não osteoporóticos. Os autores recomendaram o treinamento dos profissionais para que possam usar técnicas de avaliação específicas e deste modo detectar mudanças radiográficas significativas, especialmente no nível inferior do córtex mandibular, para ajudar a identificar mulheres pacientes na pós-menopausa com baixa densidade mineral óssea, bem como fraturas vertebrais não detectadas (Mesquita, 2010).

A tomografia computadorizada (Figura 5) também pode ser utilizada, ela ajuda a determinar a porcentagem de calcificação que pode ser usada no planejamento do tratamento e prever a progressão da doença periodontal antes da perda clínica de inserção (Bezerra, 2017).

Figura 5. Tomografia computadorizada (reformatações multiplanares e reconstrução do volume total escaneado.

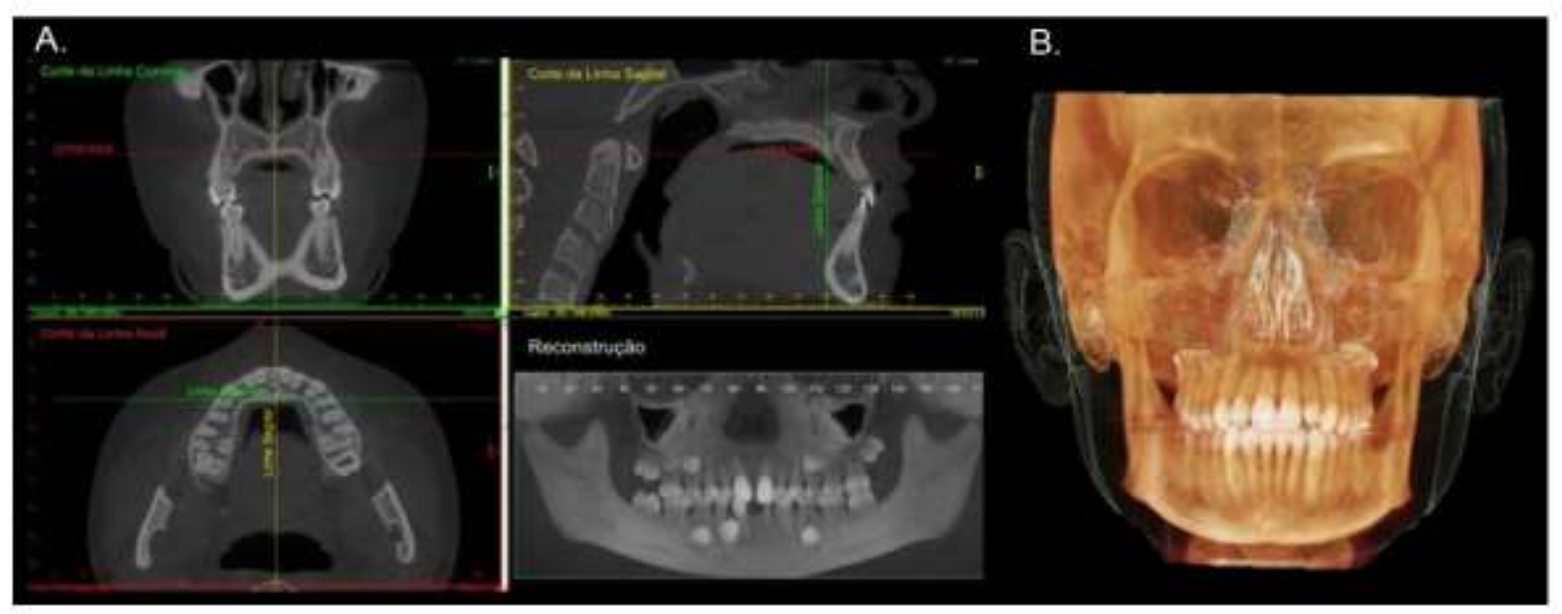

Fonte: Bezerra (2017).

Atualmente o dentista tem diversas opções para detectar previamente a osteoporose e assim determinar a melhor terapia, ou seja, a melhor forma de lidar-se com a osteoporose é através do tratamento preventivo, possibilitando a minimização de danos, tendo o diagnóstico precoce como papel vital, inclusive em âmbito odontológico, pois a osteoporose quando é detectada precocemente pode ser tratada, tendo como foco limitar seu desenvolvimento (Nascimento, et al., 2021).

\subsection{Os principais tratamentos para osteoporose}

Segundo Maxuel et al., (2020), numa primeira fase, o médico tratará uma possível deficiência de vitamina D e/ou cálcio e convidará o paciente a adotar bons hábitos alimentares e comportamentais para prevenir a osteoporose ou a sua evolução (dieta rica em cálcio e vitamina $\mathrm{D}$, cessação do tabagismo, prevenção de quedas, atividade física etc.). Se a doença for comprovada, o 
médico pode prescrever medicamentos diferentes, dependendo da situação do paciente, porém os mais comumente usados são: Bisfosfonatos, Raloxifeno, Ranelato de estrôncio, Teriparatida e Denosumab.

Tanto os sintomas da osteoporose quanto o uso de alguns medicamentos causam a desmineralização óssea nos dentes, portanto além de seu efeito no esqueleto em geral, a osteoporose também tem consequências significativas para a saúde bucal. Ao nível dos dentes, pode causar um afrouxamento gradual da dentição e a criação de espaços inter dentários. Nos maxilares, a perda óssea (figura 4) pode dificultar a colocação de implantes dentários ou próteses, como dentaduras. Da mesma forma, a doença periodontal tende a progredir mais rapidamente em pacientes com osteoporose devido à baixa densidade mineral óssea (Zavanelli, et al., 2011).

Figura 6. A) Prótese sobre implante retirada da paciente em corpo único, em razão da grande perda óssea peri-implantar. B) Presença de tecido ósseo necrótico na superfície dos implantes.
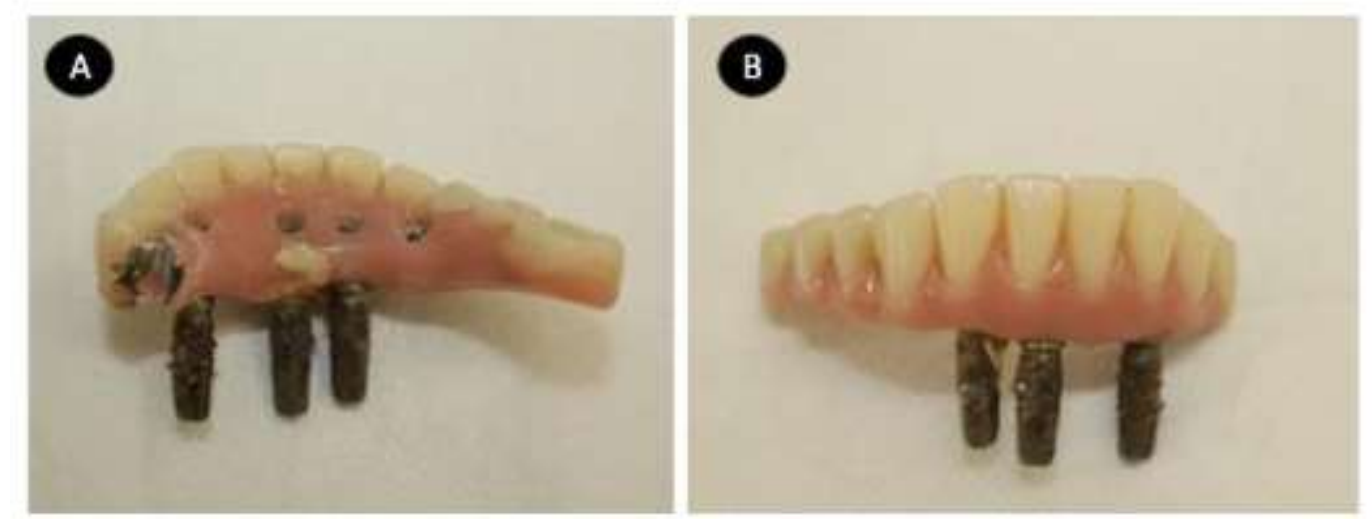

Fonte: Coléte et al. (2019).

Os tratamentos para a osteoporose podem ter consequências graves. Este é particularmente o caso dos bisfosfonatos. Esse tipo de molécula é usado no tratamento da osteoporose para ajudar a proteger e fortalecer os ossos. O maior problema vem de alguns tratamentos: os bifosfonatos que podem causar complicação grave da osteonecrose da mandíbula (Maxuel, et al., 2020).

Apesar dos bisfosfonatos serem derivados quimicamente estáveis do pirofosfato inorgânico (PPi) e quimicamente semelhantes, existem algumas diferenças que lhe conferem propriedades específicas. A estrutura de P-C-P básica dos bisfosfonatos permite muitas variações possíveis, alterando as duas cadeias laterais sobre o átomo de carbono. Diferentes substitutos ligados ao carbono central dão características únicas para cada fármaco (Figura 7). O grupo R1 fornece a afinidade dos bisfosfonatos pelos cristais de cálcio, enquanto o grupo R2 é responsável pela potência e atividade biológica nas células alvo (osteoclastos) (Biscaro, 2012). 
Figura 7. Estrutura química dos bisfosfonatos. Carbono central com dois grupos fosfatos ligados a cada um de seus lados. Esta estrutura permite uma grande biodisponibilidade dos BF's para a estrutura óssea. O grupo R1 permite maior afinidade à hidroxiapatita e o grupo R2 aumenta a potência da capacidade antirreabsortiva.

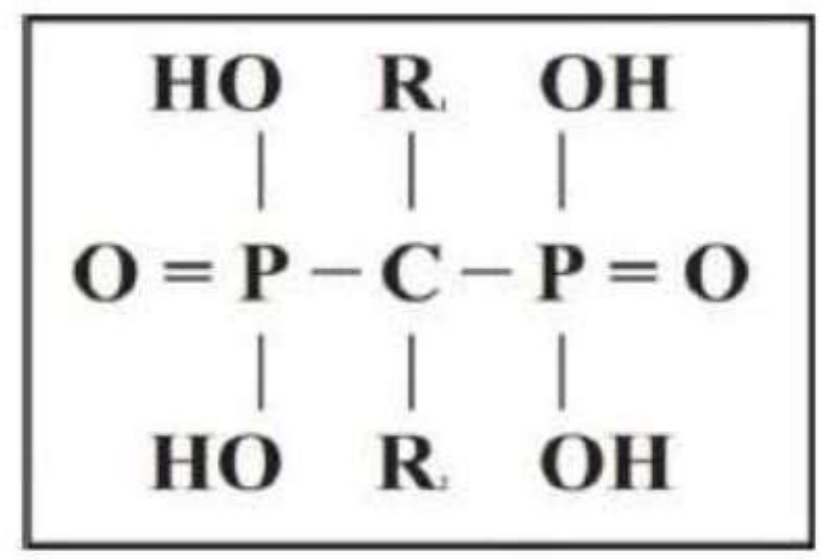

Fonte: Biscaro (2012).

Como já salientada, além dos tratamentos, a osteoporose em si pode ter consequências nos dentes, causando perda óssea na mandíbula que pode dificultar a colocação de implantes dentários ou dentaduras e próteses. Também é possível observar um afrouxamento dos dentes ou a criação de espaços interdentais. Vários estudos estabeleceram uma ligação entre a doença periodontal e a osteoporose. Um estudo conduzido na Universidade de Nova York em Buffalo em 1995 descobriu que mulheres na pós-menopausa com osteoporose tinham $86 \%$ mais chances de desenvolver doença periodontal também. A causa é a deficiência de estrogênio e baixa densidade óssea (Coléte, et al., 2019).

No entanto, muitos dentistas também desaconselham o tratamento da osteoporose, alguns acreditam que os bifosfonatos usados em pessoas com osteoporose e os bifosfonatos que podem ser prescritos para pessoas muito mais doentes com câncer ósseo. Semelhantes em ambos os casos, mas a quantidade muito maior na área de metástases ósseas. A crença de alguns reside no fato de que o verdadeiro problema da osteonecrose da mandíbula só surge quando o indivíduo sofre de metástases ósseas, nesse momento, o risco é bem maior (Brozoski, et al., 2012).

\subsubsection{Osteoporose, bifosfonatos e risco de osteonecrose da mandíbula}

Para Costa (2019), deve-se notar que certos tratamentos para osteoporose, incluindo bifosfonatos, têm sido associados a casos de osteonecrose da mandíbula, às vezes resultando em fraturas espontâneas, especialmente ao redor de implantes. Considera-se que os fatores agravantes da ingestão de bifosfonatos para osteonecrose da mandíbula são:

- Ingestão por via venosa

- Tratamento prolongado ao longo do tempo

- Uma infecção

- Combinação com corticoterapia ou quimioterapia

- Exodontias traumáticas

- Próteses ou implantes inadequados

- Doença periodontal

- Diabetes

- Consumo de álcool e fumo

- Má higiene oral 
De qualquer forma, qualquer que seja o tipo de tratamento, deve ser devidamente informado ao dentista o uso de medicamentos para a osteoporose para que ele possa oferecer os tratamentos mais adequados à cada situação. Além disso, é aconselhável realizar o tratamento odontológico necessário antes de iniciar o tratamento com bifosfonatos, exceto quando o risco de fratura for tal que não seja possível atrasar o início do tratamento.

O tratamento da osteoporose, cujo objetivo é reduzir a ocorrência de fraturas por fragilidade óssea, requer medidas preventivas não medicamentosas, somente quando não possível, recomenda-se o tratamento medicamentoso devidamente acompanhado por orientação médica. Apenas pacientes com alto risco de fratura (T score menor que -3 na densitometria óssea) ou de recorrência da $1^{\text {a }}$ fratura devem ser tratados. A quantidade insuficiente de vitamina $\mathrm{D}$ reduz a eficácia do medicamento. Portanto, é importante corrigi-lo antes de iniciar o tratamento, continuando a manter a suplementação de vitamina D (Almeida, 2019).

Os bifosfonatos são frequentemente usados primeiro, às vezes após a terapia de reposição hormonal e/ou Raloxifeno. Eles retardam a destruição óssea inibindo a atividade dos osteoclastos, aumentam a massa óssea e reduzem o risco de fraturas por osteoporose em cerca de 50\%, não só da vértebra, mas também nos idosos, do colo do útero. São eles o alendronato e o risedronato: um comprimido por semana para ser retirado da ingestão alimentar, em pé ou sentado, com um copo grande de água fracamente mineralizado. Não se deve deitar após tomar o medicamento para evitar o risco de erosão esofágica. Risedronato também é prescrito como um comprimido 2 dias consecutivos por mês (Izquierdo, et al., 2011).

O alendronato é atualmente combinado com a vitamina D3. A combinação de bisfosfonato - vitamina D (5.600 UI de vitamina D3 por semana, ou o equivalente a 800 UI por dia) pode representar uma solução de escolha, desde que, claro, a adesão ao tratamento seja boa. Aconselha-se também favorecer a ingestão de alimentos ricos em cálcio: laticínios, água altamente mineralizada (Almeida, 2019).

O zoledronato (injeção de $5 \mathrm{mg}$ ) é administrada como uma infusão intravenosa única muito lenta uma vez por ano, com o efeito colateral sem consequência clínica sendo a síndrome semelhante à gripe, que deve ser evitada. É contraindicado na insuficiência renal. Alguns estudiosos consideram a osteonecrose da mandíbula um risco muito baixo, a frequência dessa complicação do tratamento da osteoporose com bisfosfonatos de baixa dosagem é muito difícil de determinar, na faixa de um em 10.000 a 100.000 pacientes. Na verdade, é observada sobretudo em pacientes tratados por um período prolongado e em altas doses com um bifosfonato injetado por via intravenosa no contexto de patologias malignas: metástases ósseas (câncer de mama etc.) ou mieloma (Moraes, et al., 2013).

Isso envolve a exposição do osso necrótico por isquemia (redução do fluxo sanguíneo local) com o risco de superinfecção que não cicatriza após 8 semanas de desenvolvimento, afetando mais frequentemente a mandíbula, a dor é o principal sintoma, mas pode estar ausente. Certos diagnósticos diferenciais devem ser excluídos: cárie profunda, osteíte, tumor ósseo e, claro, necrose após radioterapia craniofacial. Os principais fatores de risco para osteonecrose da mandíbula são a via venosa (a mais tóxica), a duração prolongada do tratamento, infecção, combinação com corticoide ou quimioterapia, extrações dentárias traumáticas, próteses removíveis, doença periodontal, diabetes, tabagismo e má higiene oral Moraes et al., 2013).

O paciente deve ser informado do risco de necrose da mandíbula, a fim de motivá-lo para uma perfeita higiene dental e acompanhamento regular pelo cirurgião-dentista pelo menos uma vez ao ano. Antes de iniciar o tratamento, é necessário um exame bucal completo (clínico e radiográfico) e os devidos cuidados visando atingir um estado dentário saudável (Biscaro, et al., 2012).

Em pacientes tratados com bifosfonatos, o tratamento dentário ou periodontal pode ser realizado sem restrições. Quando as possibilidades do cuidado conservador são ultrapassadas, a extração dentária ou cirurgia oral pode ser realizada, mas da forma menos traumática possível, com sutura das bordas para obtenção de cicatrização de primeira linha, bochechos com clorexidina duas vezes ao dia e cobertura com antibiótico (Fontenele, et al., 2017). 
Mediante o exposto fica-se então a questão, como responder aos pacientes com osteoporose, tratados com bifosfonatos e que requerem a colocação de implantes dentários, é possível? Sim, é possível; mas após verificação e suplementação de vitamina $\mathrm{D}$, sempre com cautela, com prescrição de colutório de clorexidina e antibióticos até a cicatrização completa, monitoramento prolongado e acompanhamento regular (duas vezes ao ano) para detecção de qualquer peri-patologia. É contraindicado em pacientes tratados com bifosfonatos intravenosos para uma patologia maligna (Giro, et al., 2020).

O tratamento da osteonecrose será em primeiro lugar preventivo com a eliminação de todos os focos infecciosos dentais. Quando a osteonecrose está estabelecida, antibioticoterapia contínua, bochechos e possivelmente cirurgia com remoção de sequestros ósseos deve ser prescritos (Gonçalves, et al., 2020).

\subsection{O implante dentário e risco de ocorrência da OM.}

Vários estudos retrospectivos e séries de casos têm demonstrado associação entre a colocação de implantes e a ocorrência de osteonecrose. Assim, o risco de desenvolver OM é significativamente maior em pacientes com doenças malignas tratados com BPs intravenosos. Estudos sobre a colocação de implantes em pacientes tratados com BPs orais e sem malignidade não mostram resultados desfavoráveis em relação aos obtidos em pacientes não tratados com BPs (Fontenele, et al., 2017).

No estudo de Brozoski et al., (2012, p. 268) os autores afirmam que,

Existe uma relação exponencial direta entre o tempo de uso dos BFs e o tamanho da exposição óssea. Recomenda-se que os pacientes com valores de CTx inferiores a $150 \mathrm{pg} / \mathrm{mL}$ entrem em contato com o médico que os assiste e que seja considerada a possibilidade de suspensão da droga por um período de 4 - 6 meses. Após esse período, o teste deve ser repetido, e se o valor ainda estiver abaixo de $150 \mathrm{pg} / \mathrm{mL}$ a recomendação da literatura17 é que se estenda o tempo de suspensão da droga por um período de 6 -9 meses. Quando os valores do CTx não forem superiores a $150 \mathrm{pg} / \mathrm{mL}$ e a suspensão da droga não for possível, as orientações para o paciente quanto ao risco de desenvolvimento de ONMB devem ser reforçadas. Buscar uma forma de tratamento não invasiva deve ser sempre recomendado. É importante distinguir e ressaltar que a ONMB por BFs administrados por via oral parece ser menos frequente, menos grave e responde melhor ao tratamento com suspensão da droga e desbridamento cirúrgico

O fato é que diversas organizações profissionais emitiram recomendações sobre o manejo de pacientes em tratamento com BPs e sobre a prevenção e tratamento de osteonecrose. Essas recomendações são baseadas em revisões sistemáticas da literatura e opinião de especialistas. Embora não sejam específicos da cirurgia de implante, eles podem ser extrapolados em termos de medidas preventivas e de gerenciamento de situações específicas da cirurgia de implante (Coléte, et al., 2019).

Uma das maiores recomendações profissionais recomenda entrar em contato com o médico que prescreve bisfosfonatos. É importante para os dentistas coletar um histórico médico preciso. Devem indagar sobre o tipo de tratamento prescrito, sua duração, fatores de risco para osteonecrose e a evolução previsível da patologia que indica o tratamento com BPs. Um exame bucal completo (clínico e radiológico) pelo dentista é necessário antes de iniciar o tratamento com bifosfonatos (Madrid e Sanz, 2009).

Da mesma forma, o paciente deve ser informado que o acompanhamento oral regular (pelo menos duas vezes ao ano) e medidas rigorosas de higiene oral são essenciais para detectar e tratar patologias orais o mais precocemente possível. O paciente também será informado de que deve relatar qualquer mobilidade dentária ou inflamação da mucosa gengival (dor, inchaço, calor, vermelhidão) ao dentista e também ao médico. Se a cirurgia for essencial, qualquer paciente em uso de BP deve ser alertado sobre os riscos envolvidos antes de dar o consentimento informado (Fontenele, et al., 2017).

O tratamento com bifosfonatos intravenosos é contraindicado para a colocação de implantes dentários. O dentista deve informar o paciente sobre os riscos, contraindicações e outras alternativas terapêuticas à colocação de implantes. Ele deve receber informações claras e precisas que serão registradas no prontuário médico. Nesses pacientes, uma boa higiene oral deve ser 
mantida e o acompanhamento oral realizado a cada 4 meses. Esse acompanhamento deve ser feito em colaboração com o oncologista (Amazonas, et al., 2021).

Para os pacientes que fazem uso de bisfosfonatos por via oral, a colocação de implantes não é contraindicada em pacientes tratados com bifosfonatos orais para patologia benigna. Recomenda-se a interrupção do tratamento, pelo menos 2 a 3 meses antes da cirurgia, para pacientes em uso de PA por via oral por mais de 4 anos, com ou sem corticosteroide associado, bem como para pacientes em uso de PA por via oral há menos de 4 anos. Anos e com fatores de risco (diabetes, obesidade, tabaco etc.) ou tratamento concomitante com corticosteroides até a completa cicatrização óssea e mucosa (Gonçalves, et al., 2020).

As posições quanto à suspensão temporária dos BPs e sua duração, entretanto, não são objeto de consenso. Alguns autores consideram ser impossível dizer, por um lado, que a interrupção do tratamento elimina ou reduz o risco de OM e, por outro lado, que a interrupção do tratamento influencia negativamente o metabolismo ósseo (Amazonas, et al., 2021).

$\mathrm{Na}$ literatura não há estabelecido protocolo cirúrgico único para a colocação de implantes em um paciente em uso de bifosfonatos orais. Porém, Coléte et al., (2019), ressalta que as organizações profissionais recomendam:

- Realizar higienização bucal completa antes da operação;

- Prescrever antissépticos bucais (clorexidina) antes e durante os dias seguintes à operação;

- Prescrever a profilaxia antibiótica de acordo com o risco de infecção do paciente e a patologia tratada (câncer, metástases etc.) de acordo com as recomendações da Associação Brasileira de Odontologia;

- Utilizar abordagem segmentar, quando as necessidades cirúrgicas são múltiplas, para não expor imediatamente o paciente a extensa osteonecrose;

- Evitar anestesia intraligamentar, intra-septal e intraóssea, embora discutido, nenhum dado validado contraindica o uso de vasoconstritores;

- Realizar o procedimento cirúrgico menos traumático possível (regularização de bordas ósseas vivas, suturas sem tensão etc.);

- Recomendação de monitoramento regular com manutenção para detectar precocemente e tratar, se necessário, qualquer sinal de inflamação peri-implantar.

Segundo Fontenele et al., (2017), no caso de osteonecrose secundária à colocação do implante, o tratamento é baseado no tratamento clássico da osteonecrose. O objetivo é diminuir a dor, a infecção de partes moles e reduzir a progressão da necrose óssea. O tratamento é baseado em:

- Educação do paciente para melhorar a higiene bucal;

- Enxaguatório bucal antisséptico (clorexidina) 2 vezes ao dia;

- Início do tratamento medicamentoso para a dor (analgésico);

Início do tratamento com antibióticos por 1 a 2 semanas ou até mais, se necessário. Alguns autores recomendam doxiciclina, amoxicilina, amoxicilina associada ao ácido clavulânico e clindamicina (com ou sem metronidazol). No entanto, uma amostra local pode ser coletada para identificar os germes responsáveis e validar a escolha da antibioticoterapia; a realização de um desbridamento superficial (regularização das margens ósseas para remover as irritações dos tecidos moles); acompanhamento regular do paciente; consulta do médico prescritor sobre a continuação do tratamento com bifosfonatos (Amazonas, et al., 2021).

Em caso de evolução desfavorável, recomenda-se a retirada do implante. Sequestros ósseos, bem como espinhas ósseas irritantes, devem ser removidos sem expor mais o osso saudável. Se isso não fornecer um efeito satisfatório, medidas cirúrgicas mais radicais podem ser consideradas em um ambiente cirúrgico especializado (Costa, 2019). 
A colocação de implantes dentários representa uma terapia de escolha na reabilitação de certas áreas edêntulas. Embora os BPs tenham comprovado sua eficácia no tratamento da osteoporose e de várias patologias cancerígenas, seus efeitos colaterais não são desprezíveis. Até o momento, na ausência de evidências em termos de cuidados com implantes para pacientes em uso de bisfosfonatos, apenas recomendações de consenso direcionam os profissionais para uma atitude terapêutica que consiste em: Evitar a colocação de implantes em caso de BPs por via intravenosa; Interromper os BPs três meses antes da cirurgia para pacientes com BPs orais por mais de quatro anos e aqueles com patologia concomitante (Amazonas, et al., 2021).

Giro et al., (2020), em seu trabalho afirma que osteoporose parece não ter efeito prejudicial nas taxas de falha do implante nem na porcentagem considerável de osseointegração, afirmação está baseada em ensaios clínicos randomizados (ECR), pois a maioria destes achados não demonstram semelhanças entre pessoas com ou sem osteoporose, os estudos encontram-se na tabela abaixo.

Tabela 1. Características dos estudos que avaliaram indivíduos com osteoporose que receberam implantes dentários.

\begin{tabular}{|c|c|c|c|c|c|c|}
\hline Ret. & tstude & $N^{*}$ de sujeitos & Ne de implantes & No de fathas & Acampanhamento & $\begin{array}{l}\text { Local de inaerclo do } \\
\text { implante }\end{array}$ \\
\hline Asade oches [12] & Futronpective & a & 290001 en $\mathrm{Ch}$ & gOBO/4ACTL & 2 anos & 3811 mas $/ 332$ mand \\
\hline Nsasol e col= 1201 & lietrospective & 19000 r $393 \mathrm{CT}$ & $68090 / 1445 \mathrm{Ct}$ & 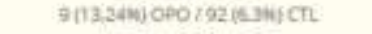 & 2 anos & 816 max/ $/ 098$ mand \\
\hline $\begin{array}{l}\text { Anorim e cols } 124 \\
1\end{array}$ & Pressective & $10000 / 20 \mathrm{ct}$ & mopor $43 \mathrm{~cm}$ & $10.5000010 \mathrm{ct}$ & 9 meser & Manobula \\
\hline \multirow[t]{2}{*}{ 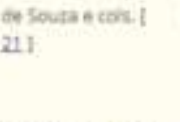 } & Autronpective & $6090 / 166 \mathrm{ch}$ & $\begin{array}{c}12(505) 1000 / 495 \text { (7) } \\
\mathrm{Cr}\end{array}$ & $12 / 50 \mathrm{~m} 1090 / 203$ copes $\mathrm{CT}$. & Nào mencianado & 356 mas / 36il mand \\
\hline & & & $\begin{array}{l}\text { Compenda beses } \\
\text { fisiolefica }\end{array}$ & com perds deses acicenal & & \\
\hline \multirow[t]{2}{*}{ Ovorak ecuis [25] } & traneversat & $\begin{array}{l}46 \text { OPO / } 16 \text { CPE / } 11 \mathrm{~s} \\
\mathrm{cT}\end{array}$ & вร่อ & 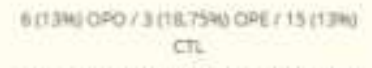 & $6 \geq 4$ anas & 432 mas/ 396 mand \\
\hline & & & Heritimplantst & 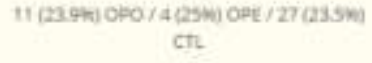 & & \\
\hline Edee e cohs. $[26]$ & $\begin{array}{l}\text { Reinto de } \\
\text { case }\end{array}$ & $10+0$ & 5 & 00 & 5 anos & Mandibuia \\
\hline Filberg a cols [22] & Aetroapectivo & $140 P O$ & 10 & $2(2.05+2)$ & 3.A anos & $38 \max / 32$ mand \\
\hline $\begin{array}{l}\text { molahun e tols. } 123 \\
1\end{array}$ & Antronoective & $\begin{array}{c}410 p 0 / 57096 / 34 \\
\mathrm{Cr}\end{array}$ & $\begin{array}{c}143000 / 197 \text { Ope } / 306 \\
\text { CT. }\end{array}$ & $10000 / 10$ ore $/ 17 \mathrm{CT}$ & 10 anes & 268 mas/ $/ 378$ mand \\
\hline
\end{tabular}

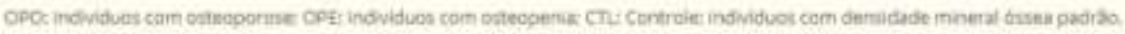

Fonte: Giro et al. (2020).

Complementarmente, o contato osso-implante de implantes dentários recuperados de mandíbulas osteoporóticas não apresentou qualquer impacto na consolidação óssea (Giro, et al., 2020).

Em pessoas com osteoporose percebe-se que o volume ósseo líquido diminuído, bem como a capacidade de carga óssea podem sofrer influência causada pela combinação dessas atividades celulares moduladas, as quais normalmente são afetadas por níveis menores de estrogênio na osteoporose pós-menopausa. Similarmente, explora-se a ideia de que após o estabelecimento da ancoragem do tecido ósseo na superfície do implante, a integração óssea é mantida. Ao contrário da remodelação óssea regular que ocorre na área trabecular, este fenômeno não é acompanhado por uma renovação ou reabsorção óssea aparente (Marco, et al., 2005; Shibli, et al., 2008).

Novamente recorrendo aos dados de Giro et al., (2020), verifica-se que o índice geral de falhas de implantes fica em torno de 10,9\%, taxa essa verificada também em estudos precedentes realizados em indivíduos sem osteopenia/osteoporose, prevalência semelhante nos casos de peri-implantite (alteração patológica dos tecidos ao redor dos implantes osseointegrados), o que sugere que doenças metabólicas pode ou não ter um impacto mínimo na patogênese da peri-implantite, ao contrário das doenças periodontais.

É preciso salientar que restaurações realizadas em implantes dentários nas mandíbulas geralmente possui a uma influência multifatorial, ou seja, sofre influências de fatores sistêmicos, fatores locais, fatores oclusais, condições periodontais, 
número e distribuição de implantes dentários no arco e forças de mordida. Outro fator a ser observado é que mesmo havendo pesquisas que apresente sucesso a longo prazo nos fatores locais e sistêmicos, ainda pouco se sabe sobre os fatores que acabam por influenciar a estabilidade dos implantes dentários posteriormente a conexão do abutment e da carga oclusal, sendo assim, os fatores endógenos na renovação e diferenciação celular é escassa. Sugere-se então que as condições sistêmicas que são associadas a pacientes com osteoporose ou osteopenia, acabam por contribuir para o agravamento da perda óssea alveolar (Coléte, et al., 2019).

Mediante o descrito até o momento observa-se que a premissa de que a inserção de implantes dentários é contraindicada em pacientes com osteoporose ou osteopenia é apenas mediada por suposições que essas patologias afetem a mandíbula na mesma proporção que afetam outras partes do corpo humano, é fato também que existe diferenças na cinética de cicatrização e na via de consolidação e remodelação óssea (Costa, 2019).

Em suma ao analisar os estudos e pesquisas sobre o tema foi possível verificar que alguns estudos demonstram a associação entre a osteoporose e taxas maiores na perda de implantes dentários, entretanto, a relação do impacto da osteoporose no contato osso-implante, possui poucas evidências que possam apoiar ou contradizer a premissa de que a osteoporose pode oferecer efeitos prejudiciais na concretização óssea, o que torna difícil apresentar conclusões definitivas sobre este impacto da osteoporose na restauração implanto suportada. É preciso parcimônia para entender essa questão, sugerindo-se assim a realização de mais estudos e pesquisas sobre o assunto. Todavia boas informações para os pacientes sobre os riscos envolvidos continuam sendo essenciais; bem como uma colaboração entre o dentista e o médico prescritor, permitirá um atendimento otimizado (Gonçalves, et al., 2020).

É primordial que devem ser tomadas as precauções necessárias ao intervir quando o paciente sofre de certas doenças e complicações adicionais: diabetes, alergias ou intolerâncias a drogas, problemas de coagulação do sangue, doenças cardiopulmonares ou renais, marca-passos etc. São os cuidados usuais na prática cirúrgica, por isso não representam grandes problemas se forem devidamente adotados (Giro, et al., 2020).

\section{Conclusão}

A osteoporose afeta pessoas idosas que, por sua vez, são as mais propensas à perda dentária, portanto, quando se submetem a tratamentos com implantes dentários, os profissionais devem fazer um estudo detalhado desses pacientes antes de prosseguir com o tratamento. Existem estudos que demonstram a influência da osteoporose na saúde bucal, mas outros indicam que as alterações na massa óssea oral não influenciam o sucesso ou o fracasso dos implantes dentários.

Ao enfraquecer a estrutura óssea e reduzir sua massa, a osteoporose pode levar ao afrouxamento dentário e complicar a colocação de implantes e próteses. Da mesma forma, tende a piorar a doença periodontal e aumentar o risco de perda do dente. Para se proteger desta doença e das suas lamentáveis consequências para os dentes, apenas uma solução, a prevenção.

Sujeitos osteoporóticos apresentaram maiores taxas de perda de implantes, entretanto, há uma menor evidência para fortalecer ou refutar a hipótese de que a osteoporose pode ter efeitos prejudiciais na consolidação óssea. Consequentemente, as conclusões finais sobre o efeito da osteoporose na terapia com implantes dentários não podem ser feitas neste momento.

\section{Referências}

Almeida, M. A. A. (2019) Papel do cálcio e vitamina D na prevenção e tratamento da osteoporose. 2019. 28 p. Revisão Tematica (Graduação em Nutrição) Universidade do Porto.

Amazonas, E. L. (2021) Atendimento Odontológico a paciente em uso de Bifosfonatos: Relato de caso. Research, Society and Development, 10(9).

Bezerra, F. V. (2017) Tomografia computadorizada de feixe cônico na identificação da baixa densidade mineral óssea: uma revisão sistemática. Dissertação Mestrado. 71p. 
Research, Society and Development, v. 10, n. 15, e82101522976, 2021

(CC BY 4.0) | ISSN 2525-3409 | DOI: http://dx.doi.org/10.33448/rsd-v10i15.22976

Biscaro, A. (2012) Influência dos bisfosfonatos na Implantodontia. 2012. 57p. Instituto Latino-Americano de Pesquisa e Ensino Odontológico.

Brozoski, M. A. et al. (2012) Osteonecrose maxilar associada ao uso de bisfosfonatos. Rev. Bras. Reumatol. 52(2):260-270.

Camargos, M. C. S., \& Bomfim, W. C. (2017) Osteoporose e Expectativa de Vida Saudável: estimativas para o brasil em 2008. Cadernos Saúde Coletiva, 25(1), 106-112.

Coléte, J. Z. et al. (2019) Implantes em pacientes com osteonecrose dos maxilares associado ao uso de bifosfonatos. Relato de caso e revisão de literatura. Arch Health Invest, 8(1). 20-27.

Costa, F. F. A. (2019) Viabilidade de implantes osteointegrados em pacientes com osteoporose. Trabalho de Conclusão de Curso. 2019. 14p. Faculdade Sete Lagoas (FASETE).

Fontenele, J. W. N. et al. (2017) Bifosfonados associados à osteonecrose dos maxilares: uma análise bibliométrica. Revista Bahiana de Odontologia.,8(4):117124.

Giro, G. et al. (2020) Impacto da osteoporose em implantes dentários: uma revisão sistemática. BJIHS, 2(5), 39-50.

Gonçalves, S. M. et al. (2020) Influência da utilização dos bifosfonatos na osseointegração dos implantes dentários. http://dx.doi.org/10.33448/rsd-v9i11.9736.

Izquierdo, C. M. et al. (2011) Terapêutica com bisfosfonatos: implicações no paciente odontológico - revisão de literatura. RFO UPF. 16(3).

Leite, A. F. et al. (2008) Radiografia panorâmica: instrumento auxiliar no diagnóstico da osteoporose. Revista Brasileira de Reumatologia. 48(4). 226-233.

Luize, D. S. et al. (2005) A influência da osteoporose na implantodontia. Arquivos em Odontologia. 41(2), 105-192.

Madrid, C., \& Sanz, M. (2009) Qual o impacto dos bifosfonatos administrados sistemicamente na terapia com implantes orais? Uma revisão sistemática. Clin Oral Implants Res. 20: 87-95.

Mahl, C. R. W. et al. (2008) Comparação de índices morfométricos obtidos na radiografia odontológica panorâmica na identificação de indivíduos com osteoporose/osteopenia. Radiologia Brasileira. 41(3). 183-187.

Marco, F., Milena, F., Gianluca, G., Vittoria, O. (2005) Osteogênese peri-implante na saúde e osteoporose. Micron. 36: 630-644.

Maxuel, F. et al. (2010) Perspectiva de tratamento da osteoporose dos maxilares por meio de fitoterápicos: Uma revisão da literatura. Revista Thêma et Scientia. $10(2)$.

Medicina Diagnóstica. Densitometria Óssea. https://www.medicinadiagnostica.com.br/exames-de-diagnostico-por-imagem/densitometria-ossea.

Mesquista, R. A. (2010) Radiografia panorâmica na avaliação da osteoporose. Trabalho de Conclusão de Curso. 2010. 47p. Faculdade de Odontologia da Universidade Federal de Minas Gerais.

Moraes, S. L. C. et al. (2013) Riscos e complicações para os ossos da face decorrentes do uso de bisfosfonatos. Rev. Bras. Odontol. 70(2).

Nascimento, T. E. (2021) Efeitos da osteoporose na cavidade bucal. Trabalho de Conclusão de Curso. Centro Universitário Uniguairacá. 30p.

Nogueira, A. S. (2017) Tomografia computadorizada de feixe cônico em implantodontia oral: Relato de série de casos. Rev. Assoc. Paul. Cir. Dent. 66(3).

Shibli, J. A., Aguiar, K. C., Melo, L., D’avila, S., Zenóbio, E. G., Faveri, M., Iezzi, G., \& Piattelli. A. (2008) Comparação histológica entre implantes recuperados de pacientes com e sem osteoporose. Int J Oral Maxillofac Surg. 37: 321-327.

Spezzia, S. (2017) Manifestações ósseas bucais da osteoporose. Rev. Ciênc. Méd., 26(2), 67-76.

Vargas, R. M., \& Schmitt, E. A. (2016) Os efeitos da osteoporose na cavidade bucal e a contribuição do cirurgião dentista: revisão de literatura. 2016. 30p. Trabalho de Conclusão de Curso. Universidade Federal de Santa Maria.

Zavanelli, R. A. Fatores locais e sistêmicos relacionados aos pacientes que podem afetar a osseointegração. RGO, Rev. gaúcha. odontol. 59(1) 